\title{
Minimal Model for Disorder-induced Missing Moment of Inertia in Solid ${ }^{4} \mathrm{He}$
}

\author{
Jiansheng $\mathrm{Wu}$ and Philip Phillips \\ Loomis Laboratory of Physics, University of Illinois at Urbana-Champaign, 1100 W.Green St., Urbana, IL., 61801-3080
}

\begin{abstract}
The absence of a missing moment inertia in clean solid ${ }^{4} \mathrm{He}$ suggests that the minimal experimentally relevant model is one in which disorder induces superfluidity in a bosonic lattice. To this end, we explore the relevance of the disordered Bose-Hubbard model in this context. We posit that a clean array ${ }^{4} \mathrm{He}$ atoms is a self-generated Mott insulator, that is, the ${ }^{4} \mathrm{He}$ atoms constitute the lattice as well as the 'charge carriers'. With this assumption, we are able to interpret the textbook defect-driven supersolids as excitations of either the lower or upper Hubbard bands. In the experiments at hand, disorder induces a closing of the Mott gap through the generation of mid-gap localized states at the chemical potential. Depending on the magnitude of the disorder, we find that the destruction of the Mott state takes place for $d+z>4$ either through a Bose glass phase (strong disorder) or through a direct transition to a superfluid (weak disorder). For $d+z<4$, disorder is always relevant. The critical value of the disorder that separates these two regimes is shown to be a function of the boson filling, interaction and the momentum cut off. We apply our work to the experimentally observed enhancement ${ }^{3} \mathrm{He}$ impurities has on the onset temperature for the missing moment of inertia. We find quantitative agreement with experimental trends.
\end{abstract}

\section{INTRODUCTION}

While superflow in a state of matter possessing a shear modulus might initially seem untenable, experimental claims for precisely this phenomenon in solid ${ }^{4} \mathrm{He}$ now abound $1,2,3,4,5,6,7,8,9,10,11,12,13$. Reported in the experiments by Kim and Chan 1.2 (KC) was a dramatic change below $200 \mathrm{mK}$ in the period of a torsional oscillator containing solid ${ }^{4} \mathrm{He}$. Because superfluids come out of equilibrium and detach from the walls of the rotated container, they are expected to give rise to a period shift in such a geometry, assuming, of course, the rotation velocity is less than the critical velocity to create a vortex. The result is a missing moment of inertia $\underline{\underline{14}, 15}$ (MMI) and hence the period of oscillation decreases. The magnitude of the MMI is a direct measure of the superfluid fraction. In the original experiments reported by Kim and Chan ${ }^{1.2}$, the superfluid fraction ranged from $.14 \%$ for ${ }^{4} \mathrm{He}$ in vycor ${ }^{1}$ to $2 \%$ in bulk ${ }^{4} \mathrm{He}$. However, Rittner and Reppy ${ }^{8,9}$ have shown that the quench time for solidifying the liquid is pivotal in determining the superfluid fraction. In one extreme, when the sample is fully annealed, no MMI occurs. In the other, the MMI increased to an astounding $20 \%$ in samples in which the solidification from the liquid occurred in less than 2 minutes. While not all groups 13 have been able to eliminate the MMI signal entirely by annealing $5,6,7$ the sample and in fact there is at least one claim of MMI in a single crystal 16 , the enhancement of MMI by a rapid quench does not seem to be in question. In fact, two independent experiments point to the key role played by disorder: 1) the Todoschenko et. al 17 measurement that the melting curve of ${ }^{4} \mathrm{He}$ remained unchanged from the $T^{4}$ law expected for phonons in ultra-pure samples with a ${ }^{3} \mathrm{He}$ concentration of $0.3 \mathrm{ppb}$ and 2) the experiments of Clark and Chan ${ }^{3}$ that increasing the ${ }^{3} \mathrm{He}$ impurity concentration ${ }^{3}$ from $20 \mathrm{ppm}$ to $40 \mathrm{ppm}$ increases the transition temperature from $0.35 \mathrm{~K}$ to $0.55 \mathrm{~K}$.

Clearly the standard textbook supersolid in which vacancy or interstitial defects Bose condense ${ }^{18.19}$ fails to explain the disorder dependence of the MMI. In fact, it is unclear at this writing even if a supercomponent is needed 20 to explain the MMI, primarily because experiments 21 designed to detect persistent mass flow have revealed no telltale signature. Monte Carlo simulations ${ }^{22}$ reveal, however, that superflow in solid ${ }^{4} \mathrm{He}$ is confined to grain boundaries. This observation is supported by the experiments of Sasaki et al $\underline{10}^{10}$ who observed mass flow only in samples containing grain boundaries. Nonetheless, the precise relationship between this experiment and the torsional oscillator measurements is unclear because mass flow was observed at temperatures (1.1K which is not far from the bulk superfluid transition temperature) vastly exceeding the onset temperature for MMI in the torsional oscillator experiments 2 , namely $T_{c}=0.2 \mathrm{~K}$.

Even if the MMI is not tied to superflow, disorder is still the key player underlying the experimental observations 13 . As disorder can induce superfluidity in the disordered Bose-Hubbard model, we explore its utility as a minimal model for the experimental observations. Certainly, this model does not have all of the microscopic details necessary to describe ${ }^{4} \mathrm{He}$, in particular the precise details needed to describe a grain boundary or the long-range interactions between ${ }^{4} \mathrm{He}$ atoms. Our central claim is only that it serves as a minimal model to describe disorder-induced superflow in a bosonic system. Our work is based on a simple claim: ${ }^{4} \mathrm{He}$ is a hexagonally close-packed self-generated Mott insulator. In a self-generated or self-assembled Mott insulator, the lattice and the 'charge carriers' are one and the same. In contrast, in Fermionic Mott insulators, the electrons occupy pre-existing lattice sites formed by the ions. Our characterization of ${ }^{4} \mathrm{He}$ as a self-generated Mott insulator is relevant for three reasons: 1) In a supersolid the relevant transport is of the ${ }^{4} \mathrm{He}$ atoms themselves. Hence, if they form a Mott insulator in the clean system, no transport is possible. 2) Experiments $\frac{13}{3}$ and simulations 
find an absence of MMI in the clean limit 22,23 . 3) We can immediately classify the candidate supersolids with this scheme because disorder can either ${ }^{24} 1$ ) self-dope the system 25,26 or 2 ) create mid-gap states 27 . The former would generate either vacancies or interstitials and hence excitations in either the lower or upper Hubbard bands. The Andreev/Lifshitz ${ }^{18}$ scenario in which vacancies or interstitials Bose condense can be thought of as arising from doping a self-generated Mott insulator. We call such a state SS1. In electronic systems, disorder is well-known to have such an effect ${ }^{25}$. We will show that SS1 does not obtain in the disordered Bose Hubbard model. Rather a superfluid state (SS2) forms from overlapping localized mid-gap states $27,28,29,30,31,32,33,34,35$. We argue that SS2 is most relevant to the experimental observations.

We establish several new results in this paper. First, we use the replica technique coupled with a renormalisation group analysis to show that weak and large disorder disrupt the Mott insulator (MI) in radically different ways. In particular, the critical value of the disorder that separates these two regimes is a decreasing function of filling. Second, in the weak disorder regime a direct transition from the superfluid (SF) to the Mott insulator is possible whereas such a transition always involves the Bose glass (BG) phase at large disorder. This result resolves the controversy $31,33,34$ surrounding when the destruction of the superfluid necessitates an intermediate Bose glass phase. Finally, we offer a quantitative test of this model by applying it to the ${ }^{3} \mathrm{He}$ enhancement of $T_{c}$. The quantitative agreement suggests that the essence of the MMI in the experiments is captured by the disordered-Bose Hubbard model.

\section{INITIAL CONSIDERATIONS}

To describe boson motion in a random potential, we adopt the site-disordered Bose-Hubbard model

$H=-t \sum_{\langle i, j\rangle}\left(b_{i}^{\dagger} b_{j}+c . c\right)+\sum_{i} \epsilon_{i} n_{i}+\frac{V}{2} \sum_{i} n_{i}\left(n_{i}-1\right)$.

In this model, $b_{i}^{\dagger}$ is the creation operator for a boson at site $i$ and $n_{i}$ is the particle number operator and $t$ and $V$ are the Josephson coupling and on-site repulsion, respectively. We also define a value $J=z t$ where $z$ is the number of nearest neighboring sites.

Though much of the theoretical work ${ }^{28,29,30,32,34,35}$ on the disordered Bose-Hubbard model has confirmed the originally proposed picture that an intermediate Bose glass localized phase disrupts the MI-SF transition, several key issues remain.

$\mathrm{P} 1$. Is there a direct MI-SF transition in the presence of disorder?

For example, several analytical treatments27.32,34,35 suggest that the Bose-glass phase completely surrounds the Mott insulating phase, making a direct transition from the MI to SF impossible. However, simulations $28,29,31$ and a renormalization group analysis 33 find that a Bose glass is absent in $d=2$ at commensurate fillings. In fact, the renormalization group analysis of Pazmandi and Zimanyi 33 lays plain that the weak and strong disorder cases are fundamentally different. Only in the strong disordered case does the Bose glass phase completely surround the Mott lobes. However, Herbut ${ }^{34}$ has also provided a convincing treatment of the large-filling limit and concluded that disorder is always relevant and destruction of the superfluid obtains through the Bose glass even in $d=2$.

P2. Do Mott insulators vanish for unbounded distributions?

Fisher, et al $\stackrel{27}{=}$ argued that no Mott insulating phases are possible when the width of the disorder exceeded $V / 2$ at $T=0$. Consequently, for unbounded distributions, Mott insulators are absent at $T=0$ and only a superfluid phase exists27. Does the same hold for finite temperature? As the distributions characterizing disorder ${ }^{36}$ in optical lattices are typically unbounded, this question must be resolved.

\section{A. Resolution}

We resolve both of the problems in this paper.

First, we show that the missing ingredients that squares these seemingly contradictory results in $\mathrm{P} 1$ are 1) dimensionality, 2) critical momentum cutoff $\Lambda_{c}$ and 3 ) a filling and interaction-dependent critical value of the disorder $\Delta_{c}$. For $\epsilon=4-(d+z)>0$, disorder is always relevant. In this case, the Mott insulating phase is destroyed and a BG obtains. This is in agreement with the work of Herbut $\underline{34}$ on the destruction of superfluidity in $d=1$ and 2 always takes place through the Bose glass. He finds that $z=1.93$, implying that $\epsilon>0$, matching our criterion for the relevance of disorder.

For systems with $\epsilon<0$, there exists a boundary in phase space separating disorder relevant and disorder irrelevant regions. For filling $m=1$, a direct transition is always allowed. For large fillings, the situation is more complicated. If the momentum cutoff (determined by the lattice constant) exceeds a critical value, $\Lambda>\Lambda_{c}$, the MI is surrounded by a BG phase and direct transition from MI to SF is forbidden as illustrated in Fig. (11). In the opposite regime, $\Lambda<\Lambda_{c}$, the strength of the disorder is the key ingredient. For the weak disorder case, $\Delta<\Delta_{c}$, a direct transition is allowed for large fillings while it is forbidden for strong disorder, $\Delta>\Delta_{c}$. These results are in accord with the RG analysis of Pazmandi and Zimanyi $\mathrm{i}^{33}$ who studied an infinite range model and found 33 that for $\epsilon<0$ a direct transition is possible. For $\epsilon>0$, they found that disorder is in general relevant except at perhaps the particle-hole symmetric point at small filling where a direct transition survives at weak disorder.

In addition, we analyse a Gaussian distribution for the 


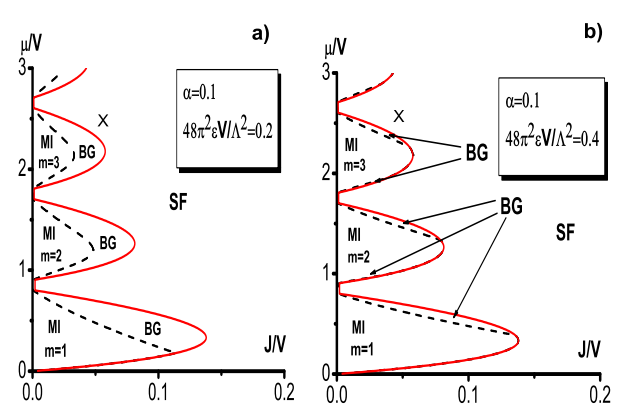

FIG. 1: Phase diagram for the disordered Bose-Hubbard model as a function of chemical potential $\mu / V$ and hopping strength, $J / V$. MI,BG and SF stands for Mott insulator, Bose glass and superfluid respectively. In the present of disorder, the lobes are shrunk, and we have two phase insider the lobes, MI and BG and outside, we have SF phase. a) The typical phase diagram when $\epsilon<0$ and $\Lambda>\Lambda_{c}$ or when $\epsilon<0, \Lambda<\Lambda_{c}$ and $\Delta>\Delta_{c}$. In this case, direct transition from MI to SF is only possible at $m=1$. b) The typical phase diagram when $\epsilon<0, \Lambda<\Lambda_{c}$ and $\Delta<\Delta_{c}$. In this case, direct transition from MI to SF is possible for many filling numbers.

site energies here and demonstrate how temperature and disorder are intertwined. At finite temperature, we establish the existence of integer-filling Mott states. However, the $T=0$ analysis is beyond the scope of the treatment here as it corresponds to the infinite disorder limit. In particular, our replica analysis on unbounded distributions is valid strictly when

$$
\beta \Delta^{2} / V<1
$$

where $\Delta$ is the variance of the distribution and $V$ the on-site repulsion. In fact, this breakdown is fundamentally related to our central point that for bosonic systems, disorder destroys Mott insulators and gives rise to superfluids. To see how this comes about, it is sufficient to integrate out the randomness by using the replica trick,

$$
\ln Z=\lim _{n \rightarrow 0} \frac{Z^{n}-1}{n}
$$

in which $n$ represents the number of replicas and $Z$ is the partition function. Performing the integral over the disorder

$$
\begin{aligned}
Z^{n} & =\prod_{i} \int d \epsilon_{i} \int D b_{i} D b_{i}^{\dagger} e^{-\frac{\left(\epsilon_{i}-(-\mu)\right)^{2}}{2 \Delta^{2}}} e^{-\beta \sum_{a} H^{a}} \\
& =\prod_{i} \int D b_{i} D b_{i}^{\dagger} e^{-\beta H_{\mathrm{eff}}} \\
H_{\mathrm{eff}} & =-t \sum_{\langle i, j>, a}\left(b_{i}^{a \dagger} b_{j}^{a}+c . c\right)-\sum_{i, a}(\mu+V / 2) n_{i}^{a} \\
& +\frac{V-\beta \Delta^{2}}{2} \sum_{i}\left(n_{i}^{a}\right)^{2}-\sum_{i, a \neq b} \frac{\beta \Delta^{2}}{2} n_{i}^{a} n_{i}^{b}
\end{aligned}
$$

results in an effective Hamiltonian for the disordered problem. Here $a$ is the replica index and we have assumed that the disorder is described by a Gaussian distribution of width $\Delta$. We see clearly that the on-site interaction is replaced by

$$
V_{\text {eff }}=V-\beta \Delta^{2} .
$$

Consequently, at sufficiently low temperature, disorder can destroy the Mott gap.

This paper is organized as follows. In the next section we compute the phase diagram for the disordered Bose Hubbard model using replicas and a renormalization group analysis. Since we start our analysis from the strongly interacting regime, any diagrams that are calculated cannot be computed using Wick's theorem. To circumvent this problem, we resorted to the analysis detailed in the Appendix. We explicitly compare the results for Gaussian and the uniform distribution case studied earlier 27 . The analysis of the phase boundary for the Bose glass is presented at the end of this section. This analysis is particularly lengthy as the topology of the phase boundaries is found to be delicately determined by the strength of the disorder and the cutoff. We close with an application of our central result that disorder enhances superfluidity to the problem of ${ }^{3} \mathrm{He}$-induced enhancement of the onset temperature for missing moment of inertia.

\section{PHASE DIAGRAM OF THE DISORDERED BOSE HUBBARD MODEL}

In this section, we derive the phase diagram for the disordered Bose-Hubbard model for the Gaussian and uniform distribution of site energies. To establish the phase boundaries for the Mott insulator (MI) and superfluid (SF) phases, we employ a saddle-point analysis on the partition function $27,38,39,40,41,42$,

$$
\begin{aligned}
Z & =Z_{0} \int \prod_{i} D \psi_{i}(\tau) D \psi_{i}^{*}(\tau) \exp \left[-S\left(\psi_{i}\right)\right] \\
S(\psi) & =\sum_{i, j}\left[J^{-1}\right]_{i j} \psi_{i}^{*}(\tau) \psi_{j}(\tau) \\
& -\sum_{i} \ln \left\langle T_{\tau} \exp \left[\int \tau \psi_{i}(\tau) b_{i}^{a \dagger}+\text { H.c. }\right]\right\rangle_{0}
\end{aligned}
$$

by introducing a Hubbard-Stratonovich field $\psi_{j}$ to release the $b_{i}^{\dagger} b_{j}$ term. Appearing in Eq. (6) are $\left[J^{-1}\right]_{i j}$, the inverse matrix of hopping rates which will determine the band structure for the kinetic energy and $Z_{0}=$ $\operatorname{Tr} \exp \left(-\beta H_{0}\right), b_{i}^{a}(\tau)=e^{H_{0} \tau} b_{i}^{a}(0) e^{-H_{0} \tau}$.

Differentiating the free energy with respect to $\psi$ yields the saddle point equation ${ }^{37}$

$$
\sum_{j}\left[J^{-1}\right]_{i j} \psi_{i}^{a}(\tau)=\left\langle b_{i}^{a}(\tau)\right\rangle
$$

Because $\psi_{i}^{a}$ is linearly related to $\left\langle b_{i}^{a}\right\rangle$, its average value will serve to define the superfluid order parameter. This 
can be seen more clearly by performing the cumulant expansion on $b_{i}^{a \dagger}(\tau)$. The action can then be rewritten as,

$$
\begin{aligned}
S(\psi) & =\beta\left[\sum_{i, a} r_{i j} \psi_{i}^{a *} \psi_{j}^{a}+c . c+u \sum_{i, a}\left|\psi_{i}^{a}\right|^{4}\right. \\
& \left.+v \sum_{i, a \neq b}\left|\psi_{i}^{a}\right|^{2}\left|\psi_{i}^{b}\right|^{2}+O\left(|\psi|^{6}\right)\right] \\
r_{i j}=\left[J^{-1}\right]_{i j} & -\delta_{i j} \int_{0}^{\beta} \int_{0}^{\beta} d \tau d \tau^{\prime}\left\langle T b_{i}^{a \dagger}(\tau) b_{i}^{a}\left(\tau^{\prime}\right)\right\rangle
\end{aligned}
$$

where $r$ matrix acts as the mass term and hence determines the appearance of superfluid phase.

\section{A. Gaussian disorder}

For the Gaussian case, the Hamiltonian consist of two parts,

$$
\begin{aligned}
& H_{0}=\frac{V_{\mathrm{eff}}}{2} \sum_{a, i}\left(n_{i}^{a}\right)^{2}-\frac{\beta \Delta^{2}}{2} \sum_{i, a \neq b} n_{i}^{a} n_{i}^{b}-\mu_{\mathrm{eff}} \sum_{a, i} n_{i}^{a} \\
& H_{1}=-t \sum_{\langle i, j\rangle} b_{i}^{a \dagger} b_{j}^{a}+c . c
\end{aligned}
$$

where $\mu_{\text {eff }}=\mu+V / 2$. Because the hopping term is a perturbation, our theory is valid strictly for $V>J$. In addition, since we are working in the limit in which the Mott lobes are well-formed, we must have that $V_{\text {eff }}>0$ and $\beta V \gg 1$. The latter two constraints can be written as $1>\alpha$ where $\alpha=\beta \Delta^{2} / V$. It is this parameter that we will use to characterize the strength of the disorder. Using the eigenstates of $H_{0}$, that is, the eigenstates of particle number, $\langle m \mid \theta\rangle=\frac{1}{2 \pi} e^{i \sum_{a} m^{a} \theta}$, we have,

$$
\begin{gathered}
\left\langle T b_{i}^{a \dagger}(\tau) b_{j}^{b}\left(\tau^{\prime}\right)\right\rangle_{0}= \\
\times \frac{1}{Z_{0}} \sum_{m}\left[\left\langle m\left|e^{H_{0} \tau} b_{i}^{a \dagger} e^{-H_{0} \tau} e^{H_{0} \tau^{\prime}} b_{j}^{b} e^{-H_{0} \tau^{\prime}}\right| m\right\rangle \theta\left(\tau-\tau^{\prime}\right)\right. \\
\left.+\left\langle m\left|e^{H_{0} \tau^{\prime}} b_{j}^{b} e^{-H_{0} \tau^{\prime}} e^{H_{0} \tau} b_{i}^{a \dagger} e^{-H_{0} \tau}\right| m\right\rangle \theta\left(\tau^{\prime}-\tau\right)\right] .
\end{gathered}
$$

For the above to be nonzero, we have to choose $a=b$ and $i=j$. Inserting a complete set of states, $1=$ $\prod_{c} \sum_{m^{c}=1}^{\infty}\left|m^{c}\right\rangle\left\langle m^{c}\right|$, between $b_{i}^{a \dagger}$ and $b_{i}^{a}$, we have only two terms left, $\left|m_{a} \pm 1(c=a) m_{c}(c \neq a)\right\rangle\left\langle m_{a} \pm 1(c=\right.$ a) $m_{c}(c \neq a) \mid$. Note that we have replica symmetry between the initial and final states. However, replica symmetry breaking must be present in the intermediate states to have a nonzero correlation. The inserted state together with the creation and annihilation operators will lead to a term of the form $E_{0}\left(m_{i}^{a} \pm 1, m_{i}^{b}\right)-E_{0}\left(m_{i}^{a}, m_{i}^{b}\right)$ where $E_{0}\left(m_{i}^{a}, m_{i}^{b}\right)$ is the eigenenergy of $H_{0}$. The explicit form for this term is

$$
\begin{aligned}
E_{0}\left(m_{i}^{a}, m_{i}^{b}\right) & =\frac{V_{\mathrm{eff}}}{2} \sum_{a, i}\left(m_{i}^{a}\right)^{2}-\frac{\beta \Delta^{2}}{2} \sum_{i, a \neq b} m_{i}^{a} m_{i}^{b} \\
& -\mu_{\mathrm{eff}} \sum_{a, i} m_{i}^{a} .
\end{aligned}
$$

After integrating over $\tau$ and $\tau^{\prime}$, we obtain,

$$
\begin{aligned}
& \int d \tau \int d \tau^{\prime}\left\langle T b_{i}^{a \dagger}(\tau) b_{j}^{b}\left(\tau^{\prime}\right)\right\rangle_{0} \\
= & \frac{(m+1)}{\varepsilon_{+}}\left(1-\frac{1}{\beta \varepsilon_{+}}\right)+\frac{m}{\varepsilon_{-}}\left(1-\frac{1}{\beta \varepsilon_{-}}\right) \\
\approx & \frac{(m+1)}{\varepsilon_{+}}+\frac{m}{\varepsilon_{-}}
\end{aligned}
$$

where we can neglect $1 / \beta \varepsilon_{ \pm}$only when the temperature is small relative to the Mott gap, that is, $k_{B} T / \varepsilon_{ \pm} \ll 1$. In the above equation, we are considering the energy of one replica, so the $m_{i}^{a} m_{i}^{b}$ term will give rise to $(n-1) m_{i}^{2}$, part of which is linear in $n$. Because we will take the limit $n \rightarrow 0$ in the end, we can neglect all the high order terms when we calculate the energy of one replica. In terms of $D=\beta \Delta^{2} / 2$, the energies $\varepsilon_{ \pm}$are defined as

$$
\begin{aligned}
\varepsilon_{ \pm} & =E_{0}\left(m_{i}^{a} \pm 1, m_{i}^{b}\right)-E_{0}\left(m_{i}^{a}, m_{i}^{b}\right) \\
& =\frac{V_{\text {eff }}}{2} \pm m\left(\frac{V_{\text {eff }}}{2}-(n-1) \frac{\beta \Delta^{2}}{2}\right) \mp \mu_{\mathrm{eff}} \\
& =\left\{\begin{array}{l}
\varepsilon_{+}(m)=m V-\mu-(m+1) D \\
\varepsilon_{-}(m)=(1-m) V+\mu+(m-1) D \text { (Gaussian) }
\end{array}\right.
\end{aligned}
$$

We defined $m$ to be the integer closest to $\mu_{\text {eff }} / V_{\text {eff }}$ because in the low temperature limit, only this term in $Z_{0}$ dominates. This holds for a system with non-conserved or commensurate particle number. For a system with conserved and incommensurate particle number, we should replace $m$ by the particle number $m_{i}$ on each site.

For the single-component case, $r$ is a scalar and we just need $r<0$ to have superfluid order. In our case, however, $r$ is a matrix which must be diagonalized. For simplicity, we consider only nearest neighbor hopping in one dimension case where $J_{i j}=t\left(\delta_{i, j+1}+\delta_{i, j-1}\right)$. The diagonal hopping matrix will be $\delta_{i j} J \cos \left(\frac{2 j \pi}{N}\right)$ with $j=0,1, \ldots, N-1$ which is the quantum number of momentum $k=2 \pi n / L$. Here $N$ is the number of sites, $L$ the system size, and $J=z t$ where $z$ is the number of nearest neighbors. Diagonalizing the hopping matrix will of course require various linear combinations of the $\psi_{i}$ fields. Such linear combinations will leave $\left\langle T b_{i}^{a \dagger}(\tau) b_{i}^{a}\left(\tau^{\prime}\right)\right\rangle_{0}$ invariant because of the $\delta_{i j}$ appearing in front. Consequently, the condition for superfluid order is,

$$
r_{i j}(n) \equiv \frac{1}{J \cos (2 n \pi / N)}-\left\langle T b_{i}^{a \dagger}(\tau) b_{i}^{a}\left(\tau^{\prime}\right)\right\rangle_{0} \leq 0 .
$$

Note superfluid order arises anytime one of the $r_{i j}^{\prime} s<0$. The phase diagrams we construct in this section correspond strictly to phases in which $\psi_{i}=0$ and $\psi_{i} \neq 0$. 
In the Bose glass section, we will make the distinction between the localized phase being gapped or ungapped.

The onset of a MI state is determined by the largest eigenvalue of $\left[J^{-1}\right]$. For a continuous band, this corresponds to $1 / J$. Consequently, the phase boundary separating $\mathrm{MI}$ and $\mathrm{SF}$ phases is given by

$$
\frac{1}{J}=\frac{(m+1)}{\varepsilon_{+}}+\frac{m}{\varepsilon_{-}}
$$

Using Eq. (15), we rewrite Eq.(17) as

$$
\begin{gathered}
m(m-1) V^{2}+V\left[(1-2 m) \mu+J+D\left(m+1-2 m^{2}\right)\right]+ \\
\mu^{2}+\mu[J+2 m D]-(m+1) D J+\left(m^{2}-1\right) D^{2}=0 .
\end{gathered}
$$

This equation describes a set of super-planes in terms of $V-\Delta-\mu$ for different $m$. For a given chemical potential, it describes the phase boundary as a function of disorder and $V$. For $m=1$, that is, one boson per site, we recover exactly $V_{c}(\Delta)$ (Eq. (36) ) as the phase line between $\mathrm{SF}$ and MI. The analogous expressions can also be derived for fixed disorder $\alpha=D / V$ but varying chemical potential $y=\mu / V$ and $x=J / V$ which reads,

$$
\begin{aligned}
y & =m-\frac{1}{2}-\frac{x}{2}-m \alpha \\
& \pm \frac{1}{2} \sqrt{(1-2 \alpha)^{2}+(4 m+2)(2 \alpha-1) x+x^{2}} .
\end{aligned}
$$

The result is shown in Fig. (2). From the figures, we see that the distance between the upper and lower boundaries of each lobe have shrunk by $2 \alpha$ and the whole lobe is shifted downward by $m \alpha$ relative to the ordered solution. As is evident, the MI phase still exists at finite temperature for the unbounded distribution. Finally, increasing disorder decreases the size of the Mott lobes. That the size of the Mott lobes shrinks with disorder has also been found in the extensive simulations of Trivedi and colleagues 29 for a uniform distribution of site energies.

\section{B. Uniform Distribution}

For completeness, we also compute the uniform distribution of site energies of width $2 \Delta$ studied in the original treatment of the disordered Bose-Hubbard problem ${ }^{27}$. Integrating over the disorder in this case is also straightforward and yields

$$
\begin{aligned}
Z^{n} & =\prod_{i} \int_{\Delta}^{\Delta} d \epsilon_{i} \frac{1}{2 \Delta} \int D b_{i} D b_{i}^{\dagger} e^{-\beta \sum_{a} H^{a}} \\
& =\prod_{i} \int D b_{i} D b_{i}^{\dagger} e^{-\beta H_{\mathrm{eff}}} \\
H_{\mathrm{eff}} & =-t \sum_{\langle i, j\rangle, a}\left(b_{i}^{a \dagger} b_{j}^{a}+c . c\right)-\sum_{i, a}(\mu+V / 2) n_{i}^{a}(19) \\
& +\frac{V}{2} \sum_{i}\left(n_{i}^{a}\right)^{2}-\frac{1}{\beta} \ln \sinh \left(\beta \Delta \sum_{a} n_{i}^{a}\right)
\end{aligned}
$$
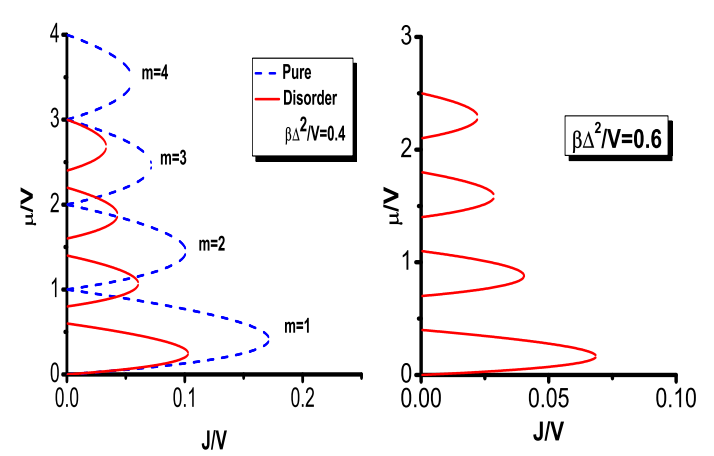

FIG. 2: Phase diagram for the disordered Bose-Hubbard model with a Gaussian distribution of site energies. The two values of the disorder correspond to $\alpha=\beta \Delta^{2} / V=0.4$ and $\alpha=0.6$.

$$
+\frac{1}{\beta} \ln \left(\beta \sum_{a} n_{i}^{a}\right),
$$

where the last two terms are interactions generated by the integration over the disorder. Note the $\beta \Delta^{2}$ reduction of the on-site repulsion is absent in the uniform distribution case. Consequently, the $T=0$ limit can be taken explicitly. Introducing $\psi_{i}^{a}$ and still choosing the basis that diagonalizes $H_{0}$ to perform the cumulant expansion, we compute the last two terms at $T=0$ and the $n \rightarrow 0$ limit to be

$$
\begin{aligned}
\lim _{\beta \rightarrow+\infty} & -\left[\frac{1}{\beta} \ln \sinh \left(\beta \Delta \sum_{a} m_{i}^{a} \pm 1\right)-\frac{1}{\beta} \ln \left(\beta \sum_{a} m_{i}^{a} \pm 1\right)\right] \\
& +\left[\frac{1}{\beta} \ln \sinh \left(\beta \Delta \sum_{a} m_{i}^{a}\right)-\frac{1}{\beta} \ln \left(\beta \sum_{a} m_{i}^{a}\right)\right] \\
& =-\lim _{\beta \rightarrow+\infty} \lim _{n \rightarrow 0} \frac{1}{\beta} \ln \left[\frac{\sinh \left(\beta \Delta n m_{i}^{a} \pm \beta \Delta\right)}{\sinh \left(\beta \Delta m_{i}^{a}\right)}\right] \\
& =-\lim _{\beta \rightarrow+\infty} \lim _{y=n \Delta m \rightarrow 0} \frac{1}{\beta} \ln \left[\frac{\sinh (y \pm \beta \Delta)}{y}\right] \\
& =-\lim _{\beta \rightarrow+\infty} \frac{1}{\beta} \ln \cosh ( \pm \beta \Delta) \\
& =-\Delta
\end{aligned}
$$

Thus we have,

$$
\begin{aligned}
& \varepsilon_{+}(m)=m V-\mu-\Delta \\
& \varepsilon_{-}(m)=(1-m) V+\mu-\Delta \text { (uniform) }
\end{aligned}
$$

where $\mu$ is replaced by $\mu+\Delta$ in the first term and by $\mu-\Delta$ in the second term. It is this structure that makes the width of the MI lobes shrink by $\Delta$ as a function of filling relative to that in the clean limit. We then use Eq. (14) to obtain

$$
y=m-\frac{1}{2}-\frac{x}{2}
$$


a)

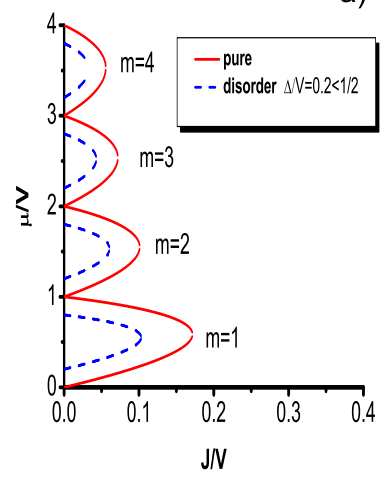

b)

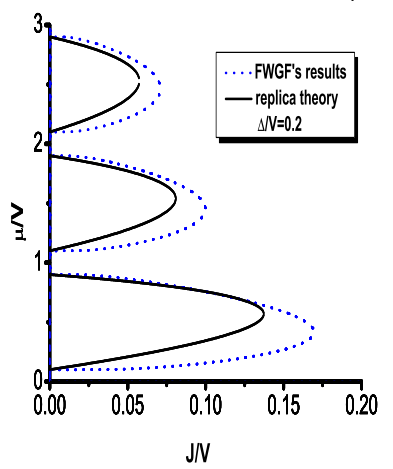

FIG. 3: a) Phase diagram for disordered Bose-Hubbard model with uniform distribution with $\delta=\Delta / V=0.4$. b) Comparison between the replica theory and the treatment of Fisher, et al ${ }^{27}$ for the infinite-range hopping model with a uniform distribution of site energies.

$$
\pm \frac{1}{2} \sqrt{(1-2 \delta)^{2}+(4 m+2)(2 \delta-1) x+x^{2}}
$$

as the phase boundary in the $x-y(J / V-\mu / V)(x$ and $y$ represent $J / V$ and $\mu / V$ he) plane for the Mott insulatorsuperfluid transition. Here $\delta=\Delta / V$.

The phase diagram in the $x-y$ plane shown in Fig. (3k) bares close resemblance to the finite temperature counterpart of the Gaussian distribution. The only difference between the two is that the disorder in the Gaussian case is characterized by $\alpha=\beta \Delta^{2}$, whereas for the uniform distribution at $T=0$, the strength of the disorder is set by $\delta=\Delta / V$. Consequently, in the uniform distribution, the Mott lobes display a vertical shift of $\delta$ rather than $\alpha$ as in the Gaussian case. For an independent check on the accuracy of the replica method, we consider the uniform distribution but with infinite range hopping. In Fig. (3b) we compare the replica method with the mean-field criterion

$$
x=-\frac{2 \delta}{\ln \left[\frac{(m-(y+\delta))^{(m+1)}(m-1-(y-\delta))^{m}}{(m-(y-\delta))^{(m+1)}(m-1-(y+\delta))^{m}}\right]}
$$

derived by Fisher, et al 27 . As is evident, only minor quantitative differences obtain, lending credence to the replica treatment presented here.

\section{Bose Glass}

In the dirty boson model, a localized phase (Bose glass) exists in which disorder rather than the on-site repulsion (Mott insulator) is the root cause. Unlike traditional spin glass phases which are characterized by an Edwards-Anderson order parameter, the Bose glass does

not admit such a description. In fact for the BoseHubbard model, the only Edwards-Anderson parameter that could be non-zero is $\left\langle b_{i}^{a}(t) b_{i}^{c}\left(t^{\prime}\right)\right\rangle$. For the superfluid phase, this order parameter is trivially nonzero. However, there is no phase in which such order exists without simultaneously relying on superfluid order. With nearest-neighbour Coulomb interactions, such a glass is possible ${ }^{43}$ independent of superfluidity. The current analysis is limited, however, solely to the on-site Coulomb case.

To analyze the Bose glass, we use the standard 27,44,45,46 one-loop renormalization group equations in conjunction with the mean-field phase boundaries to derive a criterion for the onset of the Bose glass phase. The field theory of our model is,

$$
\begin{aligned}
S(\psi) & =\sum_{k, a}\left[1-J \int d \tau\left\langle T_{\tau} b(\tau) b^{\dagger}(0)\right\rangle\right]\left|\psi^{a}(k)\right|^{2} \\
& +\sum_{k} \frac{\left(k a_{0}\right)^{2}}{2}\left|\psi^{a}(k)\right|^{2}+\sum_{i, a} g_{a a}\left|\psi_{i}^{a}\right|^{4} \\
& +\sum_{i, a \neq b} g_{a b}\left|\psi_{i}^{a}\right|^{2}\left|\psi_{i}^{b}\right|^{2}+O\left(|\psi|^{6}\right)
\end{aligned}
$$

where $a_{0}$ is the lattice constant. The coefficients $g_{a a}$ and $g_{a b}$ can be calculated using the cumulant expansion procedure outlined in the Appendix. For a Gaussian distribution, these coefficients are given by

$$
\begin{aligned}
g_{a b} & =-\frac{J^{2} \Lambda^{2}}{12 \pi^{2}}\left[\frac{(m+1)^{2}}{\varepsilon_{+}^{2}\left(\varepsilon_{+}+D / 2\right)}+\frac{m^{2}}{\varepsilon_{-}^{2}\left(\varepsilon_{-}+D / 2\right)}\right. \\
& \left.+\frac{m(m+1)}{(V-3 D)}\left(\frac{1}{\varepsilon_{+}}+\frac{1}{\varepsilon_{-}}\right)^{2}\right] \\
g_{a a} & =-\frac{J^{2} \Lambda^{2}}{48 \pi^{2}}\left[\frac{(m+1)(m+2)}{\varepsilon_{+}^{2}\left[\left(m+\frac{1}{2}\right) V-(m+2) D-\mu\right]}\right. \\
& \left.+\frac{m(m-1)}{\varepsilon_{-}^{2}\left[-\left(m-\frac{3}{2}\right) V+(m-2) D+\mu\right]}\right]
\end{aligned}
$$

The signature of the disorder-induced localized phase is the divergence of the coupling constant for the interaction between different replicas. To this end, we derive the oneloop renormalization equations $\underline{46}$

$$
\begin{aligned}
\frac{d g_{a a}}{d \xi} & =\epsilon g_{a a} \\
& -K_{d}\left((p+2) g_{a a}^{2}+p \sum_{c} g_{a c} g_{c a}\right) \\
\frac{d g_{a b}}{d \xi} & =\epsilon g_{a b}+K_{d}\left((4+2 p)\left(g_{a a}+g_{b b}\right) g_{a b}\right. \\
& \left.+4 g_{a b}^{2}+p \sum_{c} g_{a c} g_{c a}\right)
\end{aligned}
$$

for the coupling constants $g_{a b}$ and $g_{a b}$. Here $\xi$ is the standard rescaling parameter, $\epsilon=4-(d+z), K_{d}=$ 
$\frac{2}{(4 \pi)^{d / 2} \Gamma(d / 2)},\left(K_{2}=\frac{1}{2 \pi}\right), p$ is the number of the component of $\psi(p=2$ in this case) and $d$ is the spatial dimension. We are particularly interested in $p=2$ and $d=2$. Care must be taken in analyzing these equations, however, as the coupling constants, $g_{a b}$ and $g_{a a}$ are actually ultrametric matrices. Using the Parisi 47 multiplication rule for such matrices, we partition $g_{a b}$ into a diagonal part $\tilde{g}$ and an off-diagonal part which is a function $g(x)$ defined in the domain $x \in(0,1)$. At the replica symmetric fixed point, we find that

$$
\begin{aligned}
\tilde{g} & =\frac{\epsilon p}{16(p-1) K_{d}} \\
g(x) & =-\frac{\epsilon(4-p)}{16(p-1) K_{d}} \text { for } x \in[0,1] .
\end{aligned}
$$

This fixed point is unstable ${ }^{44}$ for $p>4(1-\epsilon)$. That is, for $d<3.5$, there is a runaway to the strong disorder region signalled by $g(x) \rightarrow \infty$, the signature of localization. The main criterion for the boundary to separate the disorder relevant and disorder irrelevant regions comes from the renormalization equation for $g(x)$. If we consider the replica symmetric case, we only need two parameters, the off-diagonal, $g(x)=g$, and diagonal parts, $\tilde{g}$. The renormalization equations simplify to

$$
\begin{aligned}
& \frac{d \tilde{g}}{d \xi}=\epsilon \tilde{g}-K_{d}\left[(p+2) \tilde{g}^{2}+p g^{2}\right] \\
& \frac{d g}{d \xi}=\left[\epsilon-K_{d}(4+2 p) \tilde{g}\right] g+(4-2 p) g^{2} .
\end{aligned}
$$

Two characteristic properties of the Bose glass in this RG scheme are 1) $g \rightarrow \infty$, and 2) $\psi=0$. As is well known, when $g \rightarrow+\infty$, the RG procedure breaks down. Hence, we can use the RG procedure to demarcate the boundary between the disorder relevant and disorder irrelevant regimes. From Eq. (28), the condition for $g$ to run to infinity is

$$
\epsilon-K_{d}(4+2 p) \tilde{g}>0
$$

So the boundary separating disorder relevant region and disorder irrelevant region is,

$$
\epsilon-K_{d}(4+2 p) \tilde{g}=0 .
$$

This result is in fact similar to the long wavelength limit derived by Fisher et $\mathrm{a}^{27}$. In fact, they applied the replica trick and $\mathrm{RG}$ analysis to a similar mean field action. Without considering the $p$ - dependence, they found that the coefficient of $g^{2}$ (see Eq. (28) ) is always positive. However, as is clear from Eq. (28), in the general case when the $p$-dependence is considered, this coefficient can be negative. Note the presence of $p$ is two-fold as it also generates a cross term $\tilde{g} g$ in the RG equations.

Eq. (30) together with that for $\psi=0$, that is, $r>0$ give rise to the Bose glass phase boundary in the phase diagram. This criterion depends on $\epsilon, x=J / V, y=\mu / V$, disorder and the momentum cutoff. Hence, if $\epsilon$, the disorder, and the momentum cutoff are given, Eq.(30) will define a series of curves in the $x-y$ plane. For Gaussian disorder, in the domain $\mu / V \in\left(m+\frac{1}{2}-2 \delta, m-\frac{3}{2}+\delta\right)$, $\tilde{g}<0$. As a result, if $\epsilon>0$, Eq. (29) is always satisfied, which means that for systems with $d+z<4$, disorder is always relevant. In this case, the $\psi=0$ regions all turn into the Bose glass and a direct transition between a Mott insulator and the superfluid is not possible. If $\epsilon<0$, the criterion (Eq. (29)) will separate disorder relevant and irrelevant regions in the $x-y$ plane. In general, the criterion depends on $x, y$, the disorder, $\delta$ and the momentum cutoff $\Lambda$, and is given by

$$
\begin{aligned}
x_{d} & =x_{d}(y, \Lambda, \epsilon)=\sqrt{\frac{-48 \pi^{2} \epsilon}{\Lambda^{2} / V}} \\
& \times\left\{\frac{(m+1)(m+2)}{[m-(m+1) \alpha-y]^{2}\left[m+\frac{1}{2}-(m+2) \alpha-y\right]}\right. \\
& \left.+\frac{m(m-1)}{[1-m+(m-1) \alpha+y]\left[\frac{3}{2}-m+(m-2) \alpha+y\right]}\right\}^{-1 / 2} .
\end{aligned}
$$

For different fillings $m$, we have a class of curves which form concentric lobes $x=x_{d}(y, \Lambda, \delta)$ in the $x-y$ plane. So for each filling number $m$ with $\epsilon<0$, we have a critical value $x_{d}$. If $x>x_{d}$, disorder relevant, while for $x<x_{d}$ disorder is irrelevant.

The tips of the MI lobes are at $y=(m+1) \alpha-1+(1-$ $2 \alpha) \sqrt{m(m+1)}$, precisely where $x$ reaches its maximal value. Recall $\alpha=\beta \Delta^{2} / V$. For large fillings, $m \rightarrow \infty$ and $y$ approach to $y_{0}$ where $y_{0}=m-\frac{1}{2}-m \alpha$. To see whether the disorder-relevant region lies within the MI lobes in $x-y$ plane (recalling that $x=J / V$ and $y=\mu / V)$, we calculate the value of $x, x^{\mathrm{MI}}$, for the MI lobes evaluated at $y_{0}$ and $x^{\mathrm{BG}}$ of $\mathrm{BG}$ lobes evaluated at $y_{0}$. Consequently, we consider the ratio, $\frac{x^{\mathrm{BG}}}{x^{\mathrm{MI}}}$. This ratio

$$
\frac{x^{\mathrm{BG}}}{x^{\mathrm{MI}}}=\frac{2 \pi \sqrt{6(-\epsilon)(1-2 \alpha)}}{\Lambda^{2} / V}\left(\frac{2 m+1}{\sqrt{m^{2}+m+1}}\right)
$$

is a decreasing function of filling number $m$, and for large filling number,

$$
\frac{x^{\mathrm{BG}}}{x^{\mathrm{MI}}}=\frac{4 \pi \sqrt{6(-\epsilon)(1-2 \alpha)}}{\Lambda^{2} / V} \text {, as } m \rightarrow \infty .
$$

Whether we can have a direct transition from MI to SF depends on the whether the above ratio is greater or less than one. If the above ratio is greater than one, it means that the curve demarcating the disorder-relevant region intersects the $r=0$ curve. In this case, the BG region is located in the upper and lower regions of MI lobes as depicted in Fig. (11). Consequently, in such cases, a direct transition from MI to SF is allowed. If the above ratio (Eq.(32) ) is less than one, the BG surrounds the MI and a direct transition between the MI and SF is forbidden. Because for $m=1, x_{c}$ runs to infinity as $y \rightarrow 0$, the disorder curve always intersects the $r=0$ curves at $m=1$. Consequently, we reach the conclusion that for $m=1$, 
a direct transition is always allowed. This prediction is in principle testable by direct numerical simulation. The phase diagram, Fig. (44), in the $\Delta / J-V / J$ plane displays the direct transition from the MI to the superfluid as the disorder is increased. In this plane, a further increase in the disorder leads to a transition to $m=2$ Mott insulating state. Hence, we predict that the superfluid density should be a non-monotonic function of the disorder. Similar conclusions have been reached in a Landau-Ginzburg treatment ${ }^{50}$ of the supersolid problem.

The ratio in Eq. (31) depends on the momentum cutoff $\Lambda$ and the disorder $\alpha$. We can see that increasing disorder $\alpha$ will decrease the ratio, so if $\Lambda$ is less than a critical value, no matter what strength the disorder is, the ratio is always less than one; thus direct transitions are forbidden except for $m=1$. So for a given momentum cut-off $\Lambda$ and interaction $V$, a direction transition from MI to SF is forbidden if $\Lambda<\Lambda_{c}$ where

$$
\Lambda_{c}^{4}=96 \pi^{2}(-\epsilon) V^{2}
$$

which follows from $\frac{x^{\mathrm{BG}}}{x^{\mathrm{MI}}}<1$ assuming $\alpha=0$. In this case, for any disorder strength, a direct transition is impossible between the MI and the SF at large fillings. This corroborates the result derived earlier by Herbut ${ }^{34}$ that the MI phase is always surrounded by the BG in the large-filling limit of the Bose-Hubbard model.

For large momentum cutoff $\Lambda>\Lambda_{c}$, the ratio $\frac{x^{\mathrm{BG}}}{x^{\mathrm{MT}}}$ could be greater or less than one depending on the disorder strength $\alpha$. A critical value of $\Delta_{c}$ exists. Hence, for weak disorder, $\Delta<\Delta_{c}$, we have a direct transition for large fillings $\Delta<\Delta_{c}$ with

$$
\frac{\beta \Delta_{c}^{2}}{V}=1-\frac{1}{96 \pi^{2}(-\epsilon)}\left(\frac{\Lambda^{4}}{V^{2}}\right) .
$$

which is derived from $\frac{x^{\mathrm{BG}}}{x^{\mathrm{MI}}}<1$ assuming $\Lambda>\Lambda_{c}$.

Renormalization also modifies the value of $r$ to $\underline{46}$,

$$
\begin{aligned}
r(\xi) & =r_{0} \exp \left\{\left[2-K_{d}((2+p) \tilde{g}+p g(x)] \xi\right\}\right. \\
& =r_{0} \exp \left\{-\frac{1}{2} \xi\right\}=r_{0} R_{c}^{-1 / 2} .
\end{aligned}
$$

where we have only considered the replica symmetric case. Here $R_{c}$ is the correlation length $R_{c}=\exp (\xi) \propto$ $r_{0}^{-\nu} \approx r_{0}^{-1 / 2}$, so $r(\xi) \propto r_{0}^{5 / 4}$ which means that the renormalization does not shift the MI-SF phase boundary which occurs at $r_{0}=0$.

Ultimately, it is the Bose glass that makes the disordered boson problem distinct from the disordered electronic Mott insulator. In the presence of disorder, the boson lattice adjusts (contracts or expands) so that the chemical potential remains in the gap. In the electron problem, in which the electrons occupy pre-existing lattice sites, disorder changes the position of the chemical potential ${ }^{25}$. Consequently, for the boson problem, it is the nature of the in-gap states that ultimately determines whether the disordered system is localized or not. However, as we see here the criterion is a complicated function of the system parameters.

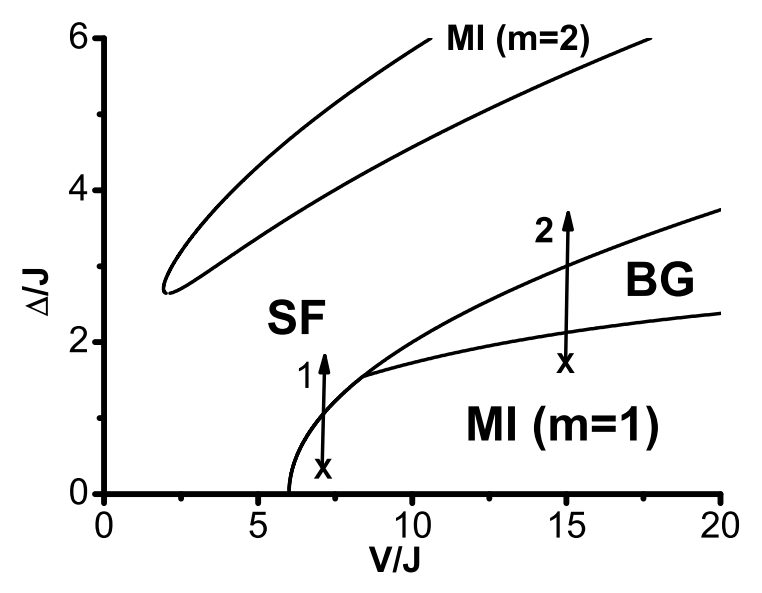

FIG. 4: A typical phase diagram in $\Delta / J-V / J$ plane for disordered Bose-Hubbard with Gaussian distributed disorder. There are three phase: MI,BG and SF. Phase at "X" which is a $\mathrm{MI}$ becomes a $\mathrm{SF}$ when the disorder is increased.

\section{D. ${ }^{3}$ He impurities}

${ }^{3} \mathrm{He}$ increase the onset temperature for the missing moment of inertia. Although we do not have a microscopic model for a grain boundary, the point defect model we have outlayed here explains this effect qualitatively as disorder can enhance the superfluid region. In essence, a disordered system with interaction $V$ can be represented by a pure system with an effective interaction $V_{\text {eff }}$. If for a pure system, the critical interaction is $V_{c}$, then for a disordered system, the corresponding critical point is $V_{\text {eff }}=V-\beta \Delta^{2}=V_{c}$. An immediate consequence is that the new boundary for the Mott-superfluid transition is shifted to higher values of the on-site interaction. That is, for the disordered system (with one boson per site) $V_{c}$ is replaced by

$$
V_{c}(\Delta)=V_{c}+\beta_{c} \Delta^{2}
$$

Consequently, to remain on the phase boundary, increasing the disorder must be compensated by an increase in the onset temperature as is seen experimentally ${ }^{3}$ for ${ }^{3}$ He defects and studied theoretically by a Abrahams and Balatsky ${ }^{50}$ using a Landau-Ginzburg approach. To formalise this, we consider ${ }^{3} \mathrm{He}$ defects with a concentration $c$ and an on-site energy $\varepsilon_{2}$. We will treat the ${ }^{4} \mathrm{He}$ atoms as having on-site energy $\varepsilon_{1}$ with concentration $1-c$. A rigorous treatment require a binomial distribution of disorder. But to get the basic scene of the influence of disorder, we use Gaussian distribution to approach this disorder. The key parameter is the variance of the distribution of onsite energies, $\Delta^{2}=\Delta_{d}^{2}+c(1-c)\left(\varepsilon_{2}-\varepsilon_{1}\right)^{2}$, where $\Delta_{d}^{2}$ is the disorder which can be eliminated by annealing. For a clean system, the transition from the Mott insulator to the superfluid is given by $k_{B} T_{c} / J=\left(V_{c}-V\right) / V_{c}^{37}$. We 


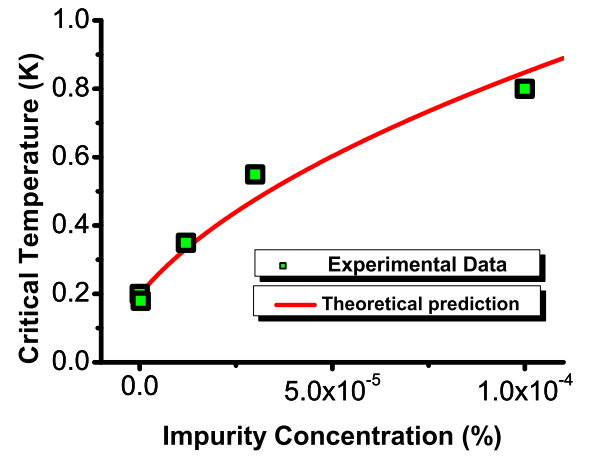

FIG. 5: Critical temperature as a function of impurity concentration. Experimental data are taken from Ref. (7).

now replace $V$ by $V_{\text {eff }}$ and solve for $T_{c}$. The solution,

$$
K_{B} T_{c}=p_{1} J+\sqrt{\left(p_{1} J\right)^{2}+p_{2} J+p_{3} J c(1-c)},
$$

has a square-root dependence with $p_{1}=\frac{\left(V_{c}-V\right)}{2 V_{c}}, p_{2}=\frac{\Delta_{d}^{2}}{V_{c}}$ and $p_{3}=\frac{\left(\varepsilon_{2}-\varepsilon_{1}\right)^{2}}{V_{c}}$. Knowing that the critical temperature is $0.2 \mathrm{~K}$ in the absence of ${ }^{3} \mathrm{He}$ impurities leads to a relationship between $p_{2} J$ and $p_{1} J$. Thus, we have two free parameters $p_{1} J$ and $p_{3} J$ to fit the curve. We show in Fig. (5D) a plot with the fitting parameters: $p_{1} J=-0.10 K$, $p_{3} J=(90 K)^{2}$ and $p_{2} J=(0.28 K)^{2}$. From the above formula, we can see that if there are no impurities and no other disorder that can be annealed away, that is, both $c=0$ and $\Delta_{d}=0$, we obtain a negative $T_{c}$ which means there is no supersolid transition. Also, if disorder is too large, $V_{\text {eff }}=V-\beta \Delta^{2}<0$, and the "net interaction" is attractive which results in an insulating phase. Consequently, for sufficiently large disorder, we also obtain an absence of a supersolid transition. Hence, although the treatment here is not rigorous, it sufficiently rich to capture the interplay between disorder, finite temperature, and supersolidity. The quantitative agreement, which is tied more to the functional form than the fitting parameters, lends credence to our claim that disorder underlies the missing moment of inertia in solid ${ }^{4} \mathrm{He}$.

\section{CONCLUSION}

We have presented what we think is the minimal model that captures disorder-induced superfluidity in bosonic systems. While we undoubtedly do not have the sufficient microscopic details to model actual grain boundaries, the results presented here offer a general framework in which the general problem of disorder-induced superfluidity can be formulated consistently. We have seen from our replica analysis and the one-loop renormalization analysis that the phase boundaries of the disordered Bose-Hubbard model can be determined but do not appear to be universal, in contrast to the phase boundaries constructed from general considerations in the early work of Fisher, et al. 27 . In particular, a direct MI-SF transition is possible as found earlier ${ }^{31.33}$; however, the criterion depends on the disorder, interaction strength and filling numbers. Further, we have shown how Mott insulating phases can be observed in unbounded distributions. This application is particularly relevant to experiments ${ }^{36}$ on optical lattices as the disorder in such systems always obeys an unbounded distribution. Since the flows are to the strong disorder limit, a treatment (currently not available) in this parameter space is essential to understanding the phase structure of the Bose-Hubbard model. Finally, because the MI phases always give rise to superfluids for intermediate disorder (for example, $0<D<V$ for Gaussian distributions), we believe this model is the correct starting point for analysing the reports of missing moment of inertia in solid ${ }^{4} \mathrm{He}$ induced by disorder, in particular the extreme sensitivity of the critical temperature to ${ }^{3} \mathrm{He}$ impurities.

\section{Acknowledgments}

We thank A. Balatsky, for initial discussions which motivated this work, D. Ceperley for critical advice, D. Huse for several conversations that shaped the final version of this paper and NSF DMR-0605769 for partial support.

\section{APPENDIX}

In the section, we will derive the effective action. The action is,

$$
\begin{aligned}
S(\psi) & =\beta \sum_{i, a} r \psi_{i}^{a *} \psi_{j}^{a}+c . c+u \sum_{i, a}\left|\psi_{i}^{a}\right|^{4} \\
& +v \sum_{i, a \neq b}\left|\psi_{i}^{a}\right|^{2}\left|\psi_{i}^{b}\right|^{2}+O\left(|\psi|^{6}\right)
\end{aligned}
$$

where in momentum space,

$$
r=\frac{1}{J \cos \left(k a_{0}\right)}-\int d \tau\left\langle T_{\tau} b(\tau) b^{\dagger}(0)\right\rangle
$$

and $a_{0}$ is the lattice constant. The coefficients $u, v$ are given by the averages,

$$
\begin{aligned}
u & =-\frac{1}{24} \int d \tau_{1} \ldots d \tau_{4}\left\langle T_{\tau} b^{a}\left(\tau_{1}\right) b^{a}\left(\tau_{2}\right) b^{a \dagger}\left(\tau_{3}\right) b^{a \dagger}\left(\tau_{4}\right)\right\rangle \\
& -\frac{1}{8}\left[\int d \tau_{1} d \tau_{2}\left\langle T_{\tau} b^{a}\left(\tau_{1}\right) b^{a \dagger}\left(\tau_{2}\right)\right\rangle\right]^{2} \\
v & =-\frac{1}{24} \int d \tau_{1} \ldots d \tau_{4}\left\langle T_{\tau} b^{a}\left(\tau_{1}\right) b^{b}\left(\tau_{2}\right) b^{a \dagger}\left(\tau_{3}\right) b^{b \dagger}\left(\tau_{4}\right)\right\rangle \\
& -\frac{1}{8}\left[\int d \tau_{1} d \tau_{2}\left\langle T_{\tau} b^{a}\left(\tau_{1}\right) b^{a \dagger}\left(\tau_{2}\right)\right\rangle\right] \\
& \times\left[\int d \tau_{3} d \tau_{4}\left\langle T_{\tau} b^{b}\left(\tau_{3}\right) b^{b \dagger}\left(\tau_{4}\right)\right\rangle\right]
\end{aligned}
$$


To compute these correlation functions, we insert a complete set of states

$$
\Pi_{i, a}\left|m_{i}^{a}><m_{i}^{a}\right|=1
$$

in between all $b^{ \pm}\left(\tau_{i}\right)$ operators and integrate over all $\tau_{i}$. The terms from the first term of the order of $u, v$ of which the order of replica indices are $a a b b$ or bbaa will cancel with the second term. So we have,

$$
\begin{aligned}
u & =-\frac{\beta}{24}\left[\frac{(m+1)(m+2)}{\epsilon_{1}^{2}\left(\epsilon_{1}+\epsilon_{2}\right)}+\frac{m(m-1)}{\epsilon_{-1}^{2}\left(\epsilon_{-1}+\epsilon_{-2}\right)}\right] \\
v & =-\beta \sum_{a \neq b}\left[\frac{(m+1)^{2}}{6 \epsilon_{1,0}^{2}\left(\epsilon_{1,0}+\epsilon_{2,1}\right)}+\frac{m(m+1)}{12 \epsilon_{1,0}^{2}\left(\epsilon_{1,0}+\epsilon_{0,1}\right)}\right. \\
& +\frac{m(m+1)}{12 \epsilon_{-1,0}\left(\epsilon_{-1,0}+\epsilon_{0,-1}\right)\left(\epsilon_{-1,0}+\epsilon_{0,-1}-\epsilon_{0,1}\right)} \\
& +\frac{m(m+1)}{12 \epsilon_{1,0}\left(\epsilon_{1,0}+\epsilon_{0,1}\right)\left(\epsilon_{1,0}+\epsilon_{0,1}-\epsilon_{0,-1}\right)} \\
& \left.+\frac{m(m+1)}{12 \epsilon_{-1,0}^{2}\left(\epsilon_{-1,0}+\epsilon_{0,-1}\right)}+\frac{m^{2}}{6 \epsilon_{-1,0}^{2}\left(\epsilon_{-1,0}+\epsilon_{-2,-1}\right)}\right]
\end{aligned}
$$

where the energies are defined as follows:

$$
\begin{aligned}
E\left(m_{i}^{a}, m_{i}^{b}\right) & =\frac{V_{\mathrm{eff}}}{2}\left(m_{i}^{a}+m_{i}^{b 2}\right)-\mu_{\mathrm{eff}}\left(m_{i}^{a}+m_{i}^{b}\right) \\
& -\frac{\beta \Delta^{2}}{2} m_{i}^{a} m_{i}^{b} \\
\epsilon_{0,-1} & =E\left(m_{i}^{a}+1, m_{i}^{b}-1\right)-E\left(m_{i}^{a}, m_{i}^{b}-1\right) \\
& =m V-(m+2) D-\mu \\
\epsilon_{1,0} & =E\left(m_{i}^{a}+1, m_{i}^{b}\right)-E\left(m_{i}^{a}, m_{i}^{b}\right)=\varepsilon_{+} \\
& =m V-(m+1) D-\mu \\
\epsilon_{2,1} & =E\left(m_{i}^{a}+1, m_{i}^{b}+1\right)-E\left(m_{i}^{a}, m_{i}^{b}+1\right) \\
& =m V-m D-\mu \\
\epsilon_{-2,-1} & =E\left(m_{i}^{a}-1, m_{i}^{b}-1\right)-E\left(m_{i}^{a}, m_{i}^{b}-1\right) \\
& =-(m-1) V+m D+\mu \\
\epsilon_{-1,0} & =E\left(m_{i}^{a}-1, m_{i}^{b}\right)-E\left(m_{i}^{a}, m_{i}^{b}\right)=\varepsilon_{-} \\
& =-\left(m_{-1}-1\right) V+(m-1) D \\
\epsilon_{0,1} & =E\left(m_{i}^{a}-1, m_{i}^{b}+1\right)-E\left(m_{i}^{a}, m_{i}^{b}+1\right) \\
& =-(m-1) V+(m-2) D+\mu \\
\epsilon_{ \pm 1} & =E\left(m_{i}^{a} \pm 1, m_{i}^{b}\right)-E\left(m_{i}^{a}, m_{i}^{b}\right)=\varepsilon_{ \pm} \\
\epsilon_{ \pm 2} & =E\left(m_{i}^{a} \pm 2, m_{i}^{b}\right)-E\left(m_{i}^{a} \pm, m_{i}^{b}\right)
\end{aligned}
$$

Denoting the momentum cutoff $\Lambda=\frac{\pi}{a_{0}}$, from above we have,

$$
\begin{aligned}
g_{a b} & =\frac{J^{2}}{a_{0}^{2}} u=-\frac{J^{2} \Lambda^{2}}{12 \pi^{2}}\left[\frac{(m+1)^{2}}{\varepsilon_{+}^{2}\left(\varepsilon_{+}+D / 2\right)}+\frac{m^{2}}{\varepsilon_{-}^{2}\left(\varepsilon_{-}+D / 2\right)}\right. \\
& \left.+\frac{m(m+1)}{(V-3 D)}\left(\frac{1}{\varepsilon_{+}}+\frac{1}{\varepsilon_{-}}\right)^{2}\right] \\
g_{a a} & =\frac{J^{2}}{a_{0}^{2}} v=-\frac{J^{2} \Lambda^{2}}{48 \pi^{2}}\left[\frac{(m+1)(m+2)}{\varepsilon_{+}^{2}\left[\left(m+\frac{1}{2}\right) V-(m+2) D-\mu\right]}\right. \\
& \left.+\frac{m(m-1)}{\varepsilon_{-}^{2}\left[-\left(m-\frac{3}{2}\right) V+(m-2) D+\mu\right]}\right]
\end{aligned}
$$

where the dependence on the cut-off is allowed.
1 E. Kim and M. H. W. Chan, Nature 427, 225 (2004).

${ }^{2}$ E. Kim and M. H. W. Chan, Science 305, 1941 (2004).

3 A. C. Clark and M. H. W. Chan, J. Low Temp. Phys. 138, 853 (2005).

${ }^{4}$ E. Kim and M. H. W. Chan, Phys. Rev. Lett. 97, 115302 (2006).
${ }^{5}$ E. Kim and M. H. W. Chan, Phys. Rev. Lett. 97, 115302 (2006).

6 A. Penzev, et al., cond-mat/0702632

7 K. Shirahama, et al., Bull. Am. Phys. Soc. 51, 450 (2006).

8 A. S. C. Rittner and J. D. Reppy, Phys. Rev. Lett. 97, 165301 (2006). 
9 A. S. C. Rittner and J. D. Reppy, Phys. Rev. Lett. 98, 175302 (2007).

10 S. Sasaki, et al. Science 313, 1098 (2006).

11 M. Kubota, et al., unpublished (2006).

12 K. Shirahama, et al., unpublished (2006).

13 For a review, see P. Phillips and A. V. Balatsky, Science 316, 1435 (2007).

14 A. J. Leggett, Phys. Rev. Lett. 25, 1543 (1970).

15 G. V. Chester and L. Reatto, Phys. Rev. 155, 88 (1967).

16 A. C. Clark, J. T. West, and M. H. W. Chan, cond-mat/0706090j.

17 I. A. Todoshchenko, H. Alles, H. J. Junes, A. Ya. Parshin, and V. Tsepelin, cond-mat/0703743

18 A. F. Andreev and I. M. Lifshitz, Sol. Phys. JETP69, 1107 (1969).

19 P. W. Anderson, W. F. Brinkman, and D. A. Huse, Science 310, 1164(2005).

20 Z. Nussinov, A.V. Balatsky, M.J. Graf, and S.A. Trugman, cond-mat/0610743, submitted to Phys. Rev. B; A.V. Balatsky, Z. Nussinov, M. Graf and S. Trugman, Phys. Rev. B 75, 094201, (2007).

21 J. Day and J. Beamish, Phys. Rev. Lett. 96, 105304, (2006).

22 E. Burovski, et al., Phys. Rev. Lett. 94, 165301 (2005); N. Profkof'ev and B. Svistunov, Phys. Rev. Lett. 94, 155302 (2005).

23 B. K. Clark and D. M. Ceperley, Phys. Rev. Lett. 93, 155303 (2004).

24 D. Huse, unpublished.

25 S. Yunoki and G. A. Sawatzky, cond-mat/0110602

${ }^{26}$ M. Boninsegni, Phys. Rev. Lett. 97, 80401 (2006).

27 M. P. A. Fisher, et al., Phys. Rev. B 40, 546 (1989).

${ }^{28}$ R. T. Scalettar, G. G. Batrouni, and G. T. Zimanyi, Phys. Rev. Lett. 66, 3144 (1991); M. Wallin, E. Sorenson, S. Girvin, and A. P. Young, Phys. Rev. B 49, 12115 (1994).

29 W. Krauth, N. Trivedi, and D. Ceperley, Phys. Rev. Lett. 67, 2307 (1991); K. Singh and D. S.Rokhsar, Phys. Rev. B 46, 3002 (1992); P. Sen, N. Trivedi, D. M. Ceperley, Phys. Rev. Lett. 86, 4092 (2001); M. Makivic, N. Trivedi, S. Ullah, ibid 71, 2307 (1993).

30 J. Freericks and H. Monien, Phys. Rev. B 53, 2691 (1996).

31 J. Kisker and H. Rieger, PRB, R11981 vol. 55 (1997).
32 R. V. Pai, et al. Phys. Rev. Lett. 76, 2937 (1996); N. Prokof'ev and B. V. Svistunov, Phys. Rev. Lett. 80, 4355 (1998); B. V. Svistunov, Phys. Rev. B 54, 16131 (1996).

33 F. Pazmandi and G. T. Zimanyi, Phys. Rev. B 57, 5044 (1998).

${ }^{34}$ I. Herbut, Phys. Rev. Lett. 79, 3502 (1997); ibid Phys. Rev. B 57, 13729 (1998).

35 M. B. Hastings, Phys. Rev. B 64, 24517 (2001).

36 B. DeMarco, private communication. In his experiments, the distribution characterising the disorder decays as an exponential rather than Gaussian. We have also analysed this case and find that our conclusions hold as well.

37 P. Phillips, Advanced Solid State Physics, (Westview, Colorado) (2003).

38 N. Read, S. Sachdev and J. Ye, Phys. Rev. B 52, 384 (1995).

39 J. Wu and P. Phillips, Phys. Rev. B 73, 214507 (2006).

40 D. Dalidovich and P. Phillips, Phys. Rev. B 59, 11925 (1999).

41 D. Dalidovich and P. Phillips, Phys. Rev. Lett. 89, 27001 (2002).

42 P. Phillips and D. Dalidovich, Phys. Rev. B 68, 104427 (2003).

43 S. Pankov and V. Dobrosavljevic, Phys. Rev. Lett. 94, 046402 (2005).

44 G. Grinstein and A. Luther, Phys. Rev. B 13, 1329 (1976).

45 T. Giamarchi, et al., Phys.Rev. B 37, 325 (1988)

46 V. Dotsenko and D. E. Feldman, J.Phys.A: Math. Gen. 28,5183-5206 (1995)

47 M. Mezard and G. Parisi, J. Phys. I 1, 809 (1991).

48 Note the renormalization group equations provide a phase boundary that intersects each lobe at $m \pm \delta$. Hence, a shortcoming of the current analysis is its inability to say anything definitive about the regions on the y-axis spanning $y \in[m+\delta, m+1-\delta]$. Such regions are governed by Griffiths singularities and appear to be beyond the scope of our analysis.

49 N. F. Mott, Metal-Insulator Transitions (Taylor \& Francis, London, 1974).

50 A. V. Balatsky and E. Abrahams, J. Sup. and Novel Mag. 19, 395 (2006). 